\title{
Congruences among generalized Bernoulli numbers
}

\author{
by \\ Janusz Szmidt (Warszawa), Jerzy Urbanowicz (Warszawa) \\ and Don ZAGier (Bonn)
}

For a Dirichlet character $\chi$ modulo $M$, the generalized Bernoulli numbers $B_{m, \chi} \in \mathbb{Q}(\chi(1), \chi(2), \ldots)(m=0,1, \ldots)$ are defined by the generating function

$$
\sum_{a=1}^{M} \frac{\chi(a) t e^{a t}}{e^{M t}-1}=\sum_{m=0}^{\infty} B_{m, \chi} \frac{t^{m}}{m !} .
$$

The main interest of these numbers is that they give the values at negative integers of Dirichlet $L$-series: if $L(s, \chi)=\sum_{n \geq 1} \chi(n) n^{-s}(\Re(s)>1)$ is the $L$-series attached to $\chi$, then we have the formula

$$
L(1-m, \chi)=-\frac{B_{m, \chi}}{m} \quad(m \geq 1) .
$$

The number $B_{0, \chi}$ equals $\varphi(M) / M$ ( $\varphi$ is Euler's phi-function) if $\chi$ is the principal character and 0 otherwise. If $m \geq 1$, then $B_{m, \chi}=0$ if $\chi(-1)=$ $(-1)^{m-1}$ (unless $M=m=1$ ). For $m>1$ the converse is also true, by (2) and the functional equation of $L(s, \chi)$, but we will not use this.

We are going to study some objects related to quadratic characters. Let $d$ be the discriminant of a quadratic field, and denote by $\chi_{d}=\left(\frac{d}{.}\right)$ the associated quadratic character (Kronecker symbol). The numbers $B_{m, \chi_{d}} / m$ are always integers unless $d=-4$ or $d= \pm p$, where $p$ is an odd prime number such that $2 m /(p-1)$ is an odd integer, in which case they have denominator 2 or $p$, respectively (cf. [3] or [6]). We also have the case $d=1$ for which $\chi_{d}$ is the trivial character; in this case, the denominator of $B_{m} / m$ contains exactly those primes $p$ for which $p-1$ divides $m$. Together, these numbers $d$ are the so-called fundamental discriminants (they can also be described as the set of square-free numbers of the form $4 n+1$ and 4 times square-free numbers not of this form) and the corresponding characters $\chi_{d}$ give all primitive quadratic characters.

1991 Mathematics Subject Classification: 11M06, 11R42. 
In the paper we find some new congruences among the values of Dirichlet $L$-series attached to quadratic characters at negative integers (or equivalently, among the numbers $B_{m, \chi_{d}} / m$ ) modulo powers of 2 or 3 . For $r \in \mathbb{Z}$ denote by $\mathcal{T}_{r}$ the set of all fundamental discriminants dividing $r$. For example, for the divisors of 24 we have $\mathcal{T}_{1}=\mathcal{T}_{2}=\{1\}, \mathcal{T}_{3}=\mathcal{T}_{6}=\{-3,1\}$, $\mathcal{T}_{4}=\{-4,1\}, \mathcal{T}_{8}=\{-8,-4,1,8\}, \mathcal{T}_{12}=\{-4,-3,1,12\}$, and $\mathcal{T}_{24}=\mathcal{T}_{8} \cup$ $\mathcal{T}_{12} \cup\{-24,24\}$. If $\chi$ is a character modulo $M$ and $d$ any non-zero integer, then for $m \geq 0$ we set

$$
B_{m, \chi}^{[d]}=\prod_{p \mid d, p \text { prime }}\left(1-\chi(p) p^{m-1}\right) \cdot B_{m, \chi}
$$

(this is just $B_{m, \chi^{\prime}}$ for the character $\chi^{\prime}$ modulo $M|d|$ induced by $\chi$, as we shall check below). Finally, we have the generalized Bernoulli polynomial defined by

$$
B_{m, \chi}^{[d]}(X)=\sum_{n=0}^{m}\left(\begin{array}{c}
m \\
n
\end{array}\right) B_{n, \chi}^{[d]} X^{m-n},
$$

which has the property $B_{m, \chi}^{[d]}(-X)=(-1)^{m} \chi(-1) B_{m, \chi}^{[d]}(X)$ unless $M=$ $m=d=1$.

TheOREM. Let $d$ be a fundamental discriminant and $r$ and $c$ be integers prime to $d$ with $r \mid 24$. Then for any $m \geq 1$ the number

$$
r^{m-1} \varphi(r) \sum_{e \in \mathcal{T}_{d}} \chi_{e}(c) B_{m, \chi_{e}}^{[d]}-\sum_{\tau \in \mathcal{T}_{r}} \chi_{\tau}(-d) \sum_{e \in \mathcal{T}_{d}} \chi_{e}(r c) B_{m, \chi_{e \tau}}^{[d]}(d)
$$

is an integer divisible by $2^{\nu+\varepsilon} r^{m-1} \varphi(r) m$, where $\nu$ denotes the number of prime factors of $d$ and $\varepsilon=1$ if $8 \mid d$ and 0 otherwise.

Proof. The proof of the theorem falls naturally into three parts.

1. If $\chi$ is a Dirichlet character modulo $M$, we define $\mathcal{L}_{\chi}(t)=\sum_{n=1}^{\infty} \chi(n) e^{n t}$. The series converges absolutely for $\Re(t)<0$. From the obvious identity

$$
\sum_{n=1}^{M} \chi(n) e^{n t}=\left(1-e^{M t}\right) \mathcal{L}_{\chi}(t)
$$

and the definition (1) we obtain the Laurent expansion

$$
\mathcal{L}_{\chi}(t)=-\sum_{n=0}^{\infty} B_{n, \chi} \frac{t^{n-1}}{n !} \quad(t \rightarrow 0) .
$$

Comparing coefficients of $t^{m-1} /(m-1)$ ! on both sides of (4) gives the identity

$$
\sum_{n=1}^{M} \chi(n) n^{m-1}=\frac{1}{m} \sum_{k=1}^{m}\left(\begin{array}{c}
m \\
k
\end{array}\right) B_{m-k, \chi} M^{k} \quad(m \geq 1)
$$


which can be used to compute the generalized Bernoulli numbers $B_{m, \chi}$ inductively and whose generalization will be the basis for the proof of the theorem.

We mention that the formula (2) for the values of the Dirichlet series $L(s, \chi)$ at negative integers follows formally from $(5)$, since if we ignore all questions of convergence then the "coefficient" of $t^{r} / r !$ in $\mathcal{L}_{\chi}(t)$ is $\sum_{n \geq 1} \chi(n) n^{r}=L(-r, \chi)$. (To prove (2) rigorously one also uses equation (5): write $\Gamma(s) L(s, \chi)$ as a Mellin transform integral $\int_{0}^{\infty} \mathcal{L}_{\chi}(-t) t^{s-1} d t$, split up the integral into $\int_{0}^{1}+\int_{1}^{\infty}$, expand the first term, and compare residues at $s=1-m$.) Note also that if the character $\chi$ is induced from a character $\chi_{1}$ modulo some divisor of $M$, then

$$
\begin{aligned}
B_{m, \chi} & =B_{m, \chi_{1}} \sum_{d \mid M} \mu(d) \chi_{1}(d) d^{m-1} \\
& =B_{m, \chi_{1}} \prod_{p \mid M}\left(1-\chi_{1}(p) p^{m-1}\right)=B_{m, \chi_{1}}^{[M]} .
\end{aligned}
$$

This follows from (2) and (an analytic continuation of) the identity $L(s, \chi)=$ $L\left(s, \chi_{1}\right) \prod_{p \mid M}\left(1-\chi_{1}(p) p^{-s}\right)$, or else from (5) and a Möbius inversion argument:

$$
\begin{aligned}
\mathcal{L}_{\chi}(t) & =\sum_{\substack{n \geq 1 \\
(n, M)=1}} \chi_{1}(n) e^{n t}=\sum_{n \geq 1} \chi_{1}(n) e^{n t} \sum_{d \mid(n, M)} \mu(d) \\
& =\sum_{d \mid M} \mu(d) \chi_{1}(d) \mathcal{L}_{\chi_{1}}(d t) .
\end{aligned}
$$

2. Now let $N$ be a multiple of $M$ and $r$ an integer prime to $N$. Then

$$
\begin{aligned}
\sum_{0<n<N / r} \chi(n) & e^{r n t} \\
& =\sum_{n>0} \chi(n) e^{r n t}-\sum_{n>0, r \mid n+N} \bar{\chi}(r) \chi(n) e^{(n+N) t} \\
& =\sum_{n=1}^{\infty} \chi(n) e^{r n t}-e^{N t} \sum_{n=1}^{\infty}\left(\frac{\bar{\chi}(r)}{\varphi(r)} \sum_{\psi} \psi(n) \bar{\psi}(-N)\right) \chi(n) e^{n t} \\
& =\mathcal{L}_{\chi}(r t)-\frac{\bar{\chi}(r)}{\varphi(r)} e^{N t} \sum_{\psi} \bar{\psi}(-N) \mathcal{L}_{\chi \psi}(t),
\end{aligned}
$$

where the sum is over all Dirichlet characters $\psi$ modulo $r$. Comparing coefficients of $t^{m-1} / m !(m \geq 0)$ on both sides and using (5), we find the 
identity

$$
\text { (6) } \begin{aligned}
m r^{m-1} \sum_{0<n<N / r} \chi(n) & n^{m-1} \\
& =-B_{m, \chi} r^{m-1}+\frac{\bar{\chi}(r)}{\varphi(r)} \sum_{\psi} \bar{\psi}(-N) B_{m, \chi \psi}(N) .
\end{aligned}
$$

3. Now specialize to the case when $r$ is a divisor of 24 . Then the group $(\mathbb{Z} / r \mathbb{Z})^{\times}$has exponent 2 , so all the characters $\psi$ are quadratic. We also restrict to quadratic characters $\chi$. Specifically, we take two coprime fundamental discriminants $K$ and $d$ and let $\chi$ range over the characters $\bmod M=|K d|$ induced by $\chi_{K e}$ with $e \in \mathcal{T}_{d}$. Multiplying both sides of (6) by $\varphi(r) \chi_{e}(c)$ for a fixed integer $c$ prime to $M$ and summing over all such characters, we find

$$
\begin{aligned}
\sum_{e \in \mathcal{T}_{d}} \chi_{e}(c)\left(-r^{m-1} \varphi(r) B_{m, \chi_{K e}}^{[d]}+\chi_{K e}(r) \sum_{\tau \in \mathcal{T}_{r}} \chi_{\tau}(-N) B_{m, \chi_{K e \tau}}^{[d]}(N)\right) \\
=m r^{m-1} \varphi(r) \sum_{\substack{0<n<N / r \\
(n, d)=1}} \chi_{K}(n) n^{m-1} \sum_{e \in \mathcal{T}_{d}} \chi_{e}(n c),
\end{aligned}
$$

and this is divisible by $m r^{m-1} \varphi(r) 2^{\nu+\varepsilon}$ because

$$
\begin{aligned}
\sum_{e \in \mathcal{T}_{d}} \chi_{e}(n c) & =\prod_{p \mid d, p>2}\left(1+\left(\frac{n c}{p}\right)\right) \cdot\left(1+\left(\frac{-4}{n c}\right)\right)_{\mathrm{if} 4 \mid d} \cdot\left(1+\left(\frac{8}{n c}\right)\right)_{\mathrm{if} 8 \mid d} \\
& \equiv 0\left(\bmod 2^{\nu+\varepsilon}\right) .
\end{aligned}
$$

To get the theorem, take $N=M=|d|$ and, if $d<0$, use the evenness or oddness of $B_{m, \chi}^{[d]}(X)$ to replace the argument $N$ of the Bernoulli polynomials by $d$.

Remarks. Since $B_{m, \chi}$ is almost always integral, as mentioned at the beginning of the paper, the essential statement of the theorem is a divisibility by a power of 2 and, if $3 \mid r$, of 3 . For example, for $r=24$ it says that the quotient of (3) by $m$ is divisible by $2^{3 m+\nu} 3^{m-1}$. These congruences are of the same general type as those of [4], [5], [8], [9] and [11]. In particular, for $r=8$ we get the congruence of [8] which is modulo $2^{3 m-1+\nu} m$, and for $r=8$ and $m=1$ or 2 we get the special cases obtained in [5] or [11]. Formulas similar to (6) appear also in [2], [7], [10] and [12].

We also make some remarks about the proof. The theorem (for $r=8$ ) was found and proved by the first two authors using a different method which required a considerably longer calculation; the third author found the simpler method of proof, presented here, during a visit to the International Banach Center in Warsaw. He thanks warmly the staff of the Center for their 
hospitality. We will say a few words about the first proof, since the starting point for it was a general and very pretty formula due to B. C. Berndt [1] that can undoubtedly be applied to many other situations of this type, namely the following "character analogue of the Poisson summation formula":

$$
\sum_{a \leq l \leq b}^{*} \chi(l) G(l)=\frac{1}{\tau(\bar{\chi})} \sum_{n=-\infty}^{\infty} \bar{\chi}(n) \int_{a}^{b} G(x) e^{2 \pi i n x / M} d x .
$$

Here $G$ is a continuous function on the interval $[a, b], \chi$ is a primitive Dirichlet character modulo $M$, and the star means that the term $\chi(l) G(l)$ is to be divided by 2 if $l=a$ or $l=b$. (To prove this identity, one can write $\chi(l)$ as $\tau(\bar{\chi})^{-1} \sum_{k=1}^{M} \bar{\chi}(k) e^{2 \pi i k l / M}$ and apply the usual Poisson summation formula to the functions $G(x) e^{2 \pi i k x / M}$.) Taking $G(x)=x^{m-1}$, after some calculations one obtains an expression for the sum on the left-hand side of (6) as a linear combination of sums of the form $\sum_{n \neq 0} \bar{\chi}(n) \zeta^{n} n^{-m}$ with $\zeta$ an $r$ th root of unity, and these can be written in turn as finite linear combinations of generalized Bernoulli numbers and polynomials, giving (6). The rest of the proof is the same.

\section{References}

[1] B. C. Berndt, Character analogues of the Poisson and Euler-Maclaurin summation formula with applications, J. Number Theory 7 (1975), 413-445.

[2] —, Classical theorems on quadratic residues, Enseign. Math. 22 (1976), 261-304.

[3] L. Carlitz, Arithmetic properties of generalized Bernoulli numbers, J. Reine Angew. Math. 202 (1959), 174-182.

[4] G. Gras, Relations congruentielles linéaires entre nombres de classes de corps quadratiques, Acta Arith. 52 (1989), 147-162.

[5] K. Hardy and K. S. Williams, A congruence relating to class numbers of complex quadratic fields, ibid. 47 (1986), 263-276.

[6] H. W. Leopoldt, Eine Verallgemeinerung der Bernoullischen Zahlen, Abh. Math. Sem. Univ. Hamburg 22 (1958), 131-140.

[7] M. Lerch, Essai sur le calcul du nombre de classes de formes quadratiques binaires aux coefficients entiers, Acta Math. 29 (1905), 333-424.

[8] J. Szmidt and J. Urbanowicz, Some new congruences for generalized Bernoulli numbers of higher orders, preprint FI94-LF05 of the Fields Institute for Research in Math. Sciences.

[9] T. Uehara, On linear congruences between class numbers of quadratic fields, J. Number Theory 34 (1990), 362-392.

[10] J. Urbanowicz, Connections between $B_{2, \chi}$ for even quadratic Dirichlet characters $\chi$ and class numbers of appropriate imaginary quadratic fields I, Compositio Math. 75 (1990), 247-270, Corrigendum: ibid. 77 (1991), 119-123.

[11] - Connections between $B_{2, \chi}$ for even quadratic Dirichlet characters $\chi$ and class numbers of appropriate imaginary quadratic fields II, ibid. 75 (1990), 271-285, Corrigendum: ibid. 77 (1991), 123-125. 
[12] J. Urbanowicz, On some new congruences for generalized Bernoulli numbers, I and II, Publ. Math. Fac. Sci. Besançon, Théorie des Nombres, Années 1990/91.

Janusz Szmidt

Don Zagier

INSTITUTE OF MATHEMATICS

MAX-PLANCK-INSTITUT FÜR MATHEMATIK MILITARY ACADEMY OF TECHNOLOGY GOTTFRIED-CLAREN-STR. 26

UL. KALISKIEGO 2 D-53225 BONN, GERMANY

01-489 WARSZAWA, POLAND

Jerzy Urbanowicz

INSTITUTE OF MATHEMATICS

POLISH ACADEMY OF SCIENCES

ŚNIADECKICH 8

00-950 WARSZAWA, POLAND

Received on 28.8.1994

and in revised form on 10.2.1995 